\author{
Prevalence of illicit drug use and associated factors \\ during pregnancy in the BRISA cohort
}

\author{
Prevalencia y factores asociados con el uso de
drogas ilegales en el embarazo en la cohorte
BRISA \\ Prevalencia y factores asociados con el uso d
drogas ilegales en el embarazo en la cohorte
BRISA \\ Prevalencia y factores asociados con el uso de
drogas ilegales en el embarazo en la cohorte
BRISA
}

\section{Prevalência e fatores associados ao uso de drogas ilícitas em gestantes da coorte BRISA}

\section{BRISA}

\begin{abstract}
Resumo
O artigo objetiva analisar a prevalência e fatores associados ao uso de drogas ilícitas na gestação. Estudo transversal, aninhado à coorte pré-natal do estudo de coorte BRISA. Utilizou-se medidas de frequência e modelo de regressão logística múltipla hierarquizada. A prevalência estimada foi de 1,45\% para o uso de drogas ilícitas, 22,32\% para o uso de bebidas alcóolicas e 4,22\% para o de cigarro. A população mostrou-se jovem, de 20-34 anos de idade (81\%), com escolaridade de 9-11 anos de estudos (75,55\%), com mais da metade das mulheres fora da população economicamente ativa (52,18\%) e na classe econômica " $C$ " (67,61\%). As gestantes apresentaram nível de estresse elevado (24,46\%), sintomas de ansiedade de moderada a intensa $(40,84 \%)$ e sintomas depressivos graves $(28,8 \%)$. Aproximadamente metade $(49,72 \%)$ das gestantes relatou ter sofrido algum tipo de violência, tendo a maioria ampla rede $(72,77 \%)$ e baixo apoio social (65,21\%). Uso de drogas lícitas, níveis altos de estresse e monoparentalidade foram os fatores independentemente associados ao uso de drogas ilícitas na gestação.
\end{abstract}

Gravidez; Drogas Ilícitas; Fatores de Risco
Priscila Coimbra Rocha 1

Maria Teresa Seabra Soares de Britto e Alves 2 Deysianne Costa das Chagas 2

Antônio Augusto Moura da Silva 2

Rosangela Fernandes Lucena Batista 2

Raimundo Antonio da Silva 2 $\begin{array}{ll}1 \text { Universidade Federal da } & \text { Correspondência } \\ \text { Bahia, Salvador, Brasil. } & \text { P. C. Rocha } \\ 2 \text { Universidade Federal do } & \text { Escola de Enfermagem, } \\ \text { Maranhão, São Luís, Brasil. } & \text { Universidade Federal da } \\ & \text { Bahia. } \\ & \text { Rua Basílio da Gama s/n, } \\ & \text { sala 43, Campus } \\ & \text { Universitário do Canela, } \\ & \text { Salvador, BA } \\ & \text { 40110-060, Brasil. } \\ & \text { pcoimbrarocha@gmail.com }\end{array}$ 


\section{Introdução}

A gestação é um período de grandes transformações na vida da mulher, causando modificações significativas em seu organismo, seu psiquismo e em seu papel sociofamiliar. Nesse contexto, o uso, o abuso e a dependência de substâncias psicoativas, por se tratar de comportamento capaz de provocar consequências físicas potencialmente graves tanto para a mãe quanto para a criança, representam uma grande preocupação para as diversas instituições e esferas da sociedade ${ }^{1,2}$.

O uso de substâncias psicoativas durante a gestação - sejam elas drogas lícitas ou ilícitas em conjunto com muitos outros fatores de riscos clássicos, como: infecções, partos múltiplos, hipertensão induzida pela gravidez, trabalho extenuante, baixo índice de massa corpórea, ganho de peso insuficiente na gravidez, reprodução assistida, colo uterino curto, intervalo interpartal curto, baixa escolaridade, raça negra e história anterior de nascimento pré-termo estão relacionados à etiologia do nascimento pré-termo ${ }^{3}$.

O uso de drogas lícitas, como álcool e cigarro, durante a gestação pode levar ao comprometimento, por vezes irreversível, da integridade da saúde da mulher e da criança. A gestante que costuma usar álcool pode ter abortamento e o feto pode apresentar lesões orgânicas e neurológicas, podendo a criança nascer com um conjunto de sinais e sintomas denominado síndrome alcoólica fetal (SAF), que é reconhecida como a maior causa de retardo mental no Ocidente 4 . Já sobre o uso do tabaco na gestação, é reconhecido que os filhos de mães tabagistas apresentam menor peso ao nascerem, além disto, este comportamento é responsável por um aumento da mortalidade fetal e neonatal, maior frequência de abortos espontâneos e malformações fetais 4 .

Drogas ilícitas como maconha, cocaína, merla e crack - são consideradas deletérias à gestante e ao feto, embora a relação de causa-efeito seja difícil de ser estabelecida. Diversos autores concordam que o uso de drogas ilícitas na gestação pode ter sérios agravos à saúde física e ao bemestar psicossocial da mulher e da criança, como aborto, prematuridade, baixo peso ao nascer e diminuição do perímetro cefálico 5,6,7 .

Na Europa, a cocaína é uma das drogas mais utilizadas por mulheres no período gestacional. Em Londres, Inglaterra em 1995, foi identificado por meio de análise urinária que o consumo de substâncias ilícitas ocorreu de forma contínua durante a gestação, com uma taxa de exposição de $10,6 \%$ no primeiro trimestre. De acordo com esse estudo, a cocaína contribuiu com 1,1\% dessa taxa. Na Espanha, em 1995, foi encontrada, por meio de análise de mecônio do recém-nas- cido, positividade para abuso de drogas de 7,9\%, dentre elas a cocaína com $4,4 \% 7$.

Nos Estados Unidos, estimativas mostram taxas crescentes do uso de cocaína durante a gestação, de 10\% em 1995 a 12,4\% em 2004 8. Ainda nos Estados Unidos, a Pesquisa Nacional sobre Uso de Drogas e Saúde (NSDUH), realizada em 2010 pelo Instituto Nacional sobre Abuso de Drogas (NIDA), relata um aumento substancial do uso de substâncias ilícitas entre mulheres grávidas. Em 2010, 4,4\% das mulheres grávidas com idades entre 15 e 44 anos relataram o consumo de drogas, evidenciando aumento quando comparado à prevalência de 3\% em 20028.

No Brasil, existem poucos dados referentes a gestantes usuárias de drogas ilícitas 5,6,7. Em 1999, em Porto Alegre (Rio Grande do Sul) foi estimada taxa de uso de cocaína em gestantes de $1,7 \%$ por entrevistas maternas e de $6 \%$ por análises toxicológicas, exame de mecônio, em estudo caso-controle sobre exposição pré-natal à cocaína e efeitos comportamentais no recém-nascido, cuja amostra foi composta 62 recém-nascidos, em um hospital terciário da rede pública do Sistema Único de Saúde (SUS) ${ }^{9}$. Nesse estudo, o uso de cocaína durante a gestação apresentou associação estatisticamente significativa com a situação de monoparentalidade - mulher sem companheiro que arca sozinha com a responsabilidade da gestação - e com menores condições socioeconômicas 9 .

Ainda no Brasil, em 2012, em Maringá (Paraná), estudo transversal com 394 gestantes assistidas em unidades básicas de saúde, identificou por meio de entrevista a prevalência de $6,09 \%$ para o uso de álcool, 9,14\% para o de cigarro, $0,51 \%$ para o de maconha e $0,51 \%$ para o uso de crack 10 .

Violência contra a mulher - seja psicológica, física ou sexual; aspectos psicossociais como estresse, ansiedade, depressão; e suporte social da mulher, como rede social e apoio social, são fatores de risco já descritos associados ao uso de drogas ilícitas na gestação 1,2

Para o entendimento dos fatores associados a esse uso é necessário um modelo conceitual que explique as relações entre os fatores a serem estudados. Partiu-se do pressuposto de existirem diferentes determinações para o consumo de drogas ilícitas. Alguns fatores podem agir direta ou indiretamente sobre o uso de drogas ilícitas, definindo diferentes níveis de hierarquia: desde os mais proximais até os intermediários e distais.

A identificação de fatores associados ao consumo de drogas ilícitas na gestação, levando em conta as diferentes influências de cada um no uso de drogas, poderá permitir intervenções específicas e relevantes para a população estudada. Nesse sentido, o objetivo do estudo foi analisar 
a prevalência e os fatores associados ao uso de drogas ilícitas na gestação no Município de São Luís, Maranhão.

\section{Métodos}

Este trabalho compõe o projeto Fatores Etiológicos do Nascimento Pré-Termo e Consequências dos Fatores Perinatais na Saúde da Criança: Coortes de Nascimento em Cidades Brasileiras (BRISA), desenvolvido pela Universidade Federal do Maranhão (UFMA) em parceria com a Universidade de São Paulo (USP), realizado em dois municípios brasileiros: São Luís, e Ribeirão Preto (São Paulo).

Trata-se de estudo transversal, descritivo e analítico, realizado no Município de São Luís, capital do Estado do Maranhão, no ano de 2010. São Luís tem uma população de 1.014.837 habitantes, sendo 375.093 mulheres em idade fértil, entre 10 e 49 anos, e população estimada de 15.259 gestantes (Departamento de Informática do SUS. Informações sobre estatísticas vitais. http://tabnet.datasus.gov.br/cgi/tabc gi.exe?ibge/cnv/popma.def, acessado em 15/ Nov/2011).

Foram utilizados dados de coorte prospectiva de 1.447 gestantes, de amostra de conveniência, e cujas mulheres foram recrutadas por ocasião de uma consulta pré-natal realizada até o 5 o mês de gravidez em hospitais e unidades de saúde, na cidade de São Luís. As mulheres foram contatadas nos serviços, que dispunham de ultrassonografia gestacional, e convidadas a comparecer ao Hospital Universitário Unidade Materno-Infantil para participar da pesquisa. Apenas as mulheres com gravidez única foram incluídas no estudo. A idade gestacional foi avaliada com base na ultrassonografia realizada com menos de 20 semanas de gestação.

Para o cálculo do tamanho amostral assumiu-se uma prevalência de uso de cocaína em uma população de mulheres não gestantes de $5,7 \%$, e uma diferença de prevalência entre grupo exposto e não exposto de $4,3 \%$, poder do teste de 80 , alfa de 0,05 , sendo proporção de 1:1 entre expostos e não expostos, resultando num total de 1.318 gestantes (659 expostos e 659 não expostos). Prevendo a possibilidade de perda de segmento e/ou não-respostas, acrescentou-se $10 \%$ a esse valor, chegando à mostra mínima de 1.449 mulheres.

Para a coleta de dados foram utilizados métodos clássicos de entrevista, com aplicação de questionário padronizado por pessoa capacitada previamente. Violência sofrida e uso de drogas ilícitas compuseram a parte do questionário au- toaplicado em razão da natureza das questões, que requerem espaço reservado e privacidade para serem respondidas.

Os questionários foram aplicados após a assinatura do Termo de Consentimento Livre e Esclarecido. As variáveis utilizadas foram organizadas em sete blocos distribuídos em três níveis. No nível estão: bloco 1, que incluiu as variáveis ocupação e escolaridade; o bloco 2, classes econômicas; o bloco 3, idade e estado civil. No nível intermediário: bloco 4, violência; bloco 5 , rede e apoio social; e bloco 6 , estresse, ansiedade e depressão. No nível proximal está o bloco 6, drogas lícitas.

A idade da gestante foi categorizada em menores de 20 anos, entre 20 e 34 e 35 anos ou mais. A classe econômica foi definida segundo o Critério de Classificação Econômica Brasil, desenvolvido pela Associação Brasileira de Empresas de Pesquisa (ABEP. Critério de Classificação Econômica Brasil, 2011. http://www.abep.org), dividindo-a em classes econômicas A/B, C e D/E. Para escolaridade, considerou-se as categorias menos de 9 anos de estudos, de 9-11 e 12 anos ou mais. A ocupação da mulher foi organizada segundo a classificação do Instituto Brasileiro de Geografia e Estatística (IBGE) em: nunca trabalhou ou trabalha, trabalho como atividade que gere remuneração financeira. Foi classificada como monoparentalidade a gestante que relatou não residir com companheiro.

Sobre as variáveis relacionadas à violência utilizou-se o Questionário de Violência da Organização Mundial da Saúde (OMS), validado e traduzido para o português 11,12, composto por 32 questões, sendo investigada: psicológica, física e sexual. Considerou-se violência ao menos uma resposta positiva a qualquer de seus itens.

Para classificar rede e apoio social utilizou-se a Escala de Rede e Apoio Social do Medical Outcomes Study (MOS), validada e traduzida, usada no Estudo Pró-Saúde 13. A escala é composta por 27 itens, os 8 primeiros correspondem às perguntas do bloco de rede social e os 19 restantes ao bloco de apoio social (DATASUS. http://tabnet. datasus.gov.br/cgi/tabcgi.exe?ibge/cnv/popma. def, acessado em 15/Nov/2011). A rede social foi categorizada em ampla ou restrita. No bloco apoio social, as respostas estavam organizadas segundo a escala de Likert com variação de 1 (nunca) a 5 (sempre). Foi calculado o escore total das 19 variáveis e considerado alto os valores acima de 66, apoio social alto, correspondendo ao segundo tercil 13 .

Acerca das características psicossociais das gestantes, os sintomas de depressão foram identificados pela Escala de Rastreamento Populacional para Depressão do Centro de Estudos Epi- 
demiológicos (Center for Epidemiologic Studies Depression Scale, CES-D). A escala foi desenvolvida para uso em populações sem história conhecida de transtorno mental e avalia a intensidade dos sintomas depressivos, tendo sido validada em português 14,15. É composta por 20 itens para avaliar a presença de humor deprimido. A entrevistada informa a frequência da ocorrência dos sintomas na última semana, utilizando uma escala que varia de 0 , "raramente" (menos de 1 dia) a 3, "durante a maior parte do dia (5-7 dias)". O escore dos 4 itens positivos é invertido e somado ao escore dos demais, totalizando um escore final que variou de 0 a 60 . Utilizou-se como ponto de corte o escore $\geq 22$ para "sintomas depressivos graves", conforme preconizado em estudo prévio 16,17 .

A presença de sintomas de ansiedade foi avaliada pela Escala de Ansiedade de Beck (Beck Anxiety Inventory, BAI), validada em português 18 . A escala é utilizada para avaliação clínica da ansiedade, mas tem se mostrado um instrumento adequado para rastreamento de indivíduos com transtornos de ansiedade na população em geral. O questionário é composto por 21 itens, que identificam os sintomas de ansiedade apresentados na última semana e quantificam sua intensidade em uma escala que varia de 0 (ausente) a 3 (severo). O escore total é alcançado pela soma dos escores apresentados em cada questão e permite a classificação do nível de ansiedade em normal (0-9 pontos), leve (10-18), moderada (19-29) e intensa (30-63 pontos) 19,20. Essa variável foi categorizada em ansiedade normal/leve e moderada/intensa.

O nível de estresse foi averiguado pela Escala de Estresse Percebido (Perceveid Stress Scale, PSS-14), um instrumento curto e de fácil aplicação, traduzido e validado para a população brasileira, que identifica situações na vida do indivíduo avaliadas como estressantes, estabelecendo níveis de intensidade. As questões são de natureza geral, podendo ser usadas em qualquer subgrupo populacional, inclusive em gestantes. É composta por 14 questões sobre a frequência em que determinados sentimentos e pensamentos ocorreram no último mês, com respostas variando de 0 (nunca) a 4 (sempre). O escore é obtido revertendo-se os escores dos itens positivos e somando-se as respostas dos 14 itens, com o escore total variando de 0 a 70 . Para a avaliação a variável foi categorizada, considerando-se alto nível de estresse percebido os escores acima do último quartil 21,22.

O consumo de drogas lícitas e ilícitas foi classificado de acordo com o uso, em sim ou não, tendo em vista que qualquer frequência ou modo de uso na gestação implica potencial fator de risco tanto para a gestante quanto para o feto, uma vez que não se tem estabelecido quantidades consideradas seguras para o consumo de drogas durante a gestação, sendo recomendada como melhor conduta a abstinência 6 . A variável consumo de drogas ilícitas referiu-se ao uso no período gestacional, somente, e a mesma mulher pode referir o uso de mais de uma droga em cada período.

Para a análise dos dados, a digitação foi realizada utilizando-se o programa Access (Microsoft Corp., Estados Unidos) e os mesmos foram analisados no programa Stata 10.0 (StataCorp LP, College Station, Estados Unidos). Inicialmente, foi realizada análise descritiva das variáveis estudadas por meio de medidas de frequência e intervalo de confiança. Para avaliar os fatores associados ao uso de drogas ilícitas foi utilizado modelo de análise de regressão logística múltipla hierarquizada, conforme modelo teórico (Figura 1).

Estimou-se odds ratio (OR) como medida de associação entre a variável resposta, uso de drogas ilícitas na gestação e as demais variáveis analisadas, adotando-se nível de significância de 0,05 . Estimaram-se modelos multivariados por níveis, agrupando os blocos, introduzindo as variáveis de uma só vez (método direto de seleção de variáveis). As variáveis com valor de $\mathrm{p} \leq 0,10$ foram mantidas no modelo de análise independentemente do nível de significância apresentado após a introdução das variáveis dos blocos subsequentes. A modelagem hierarquizada iniciou com a variável nível distal, seguida das intermediárias e proximais. No modelo final foram consideradas associadas as variáveis com valor de $\mathrm{p} \leq 0,05$.

A pesquisa atendeu aos critérios da Resolução CNS no 196/1996 e suas complementares do Conselho Nacional de Saúde, e foi submetida à análise do Comitê de Ética em Pesquisa do Hospital Universitário da Universidade Federal do Maranhão, sendo aprovada pelo Parecer Consubstanciado no 223/2009.

\section{Resultados}

Dentre as 1.447 gestantes, $81 \%$ estavam na faixa etária de 20-34 anos e 75,6\% com escolaridade de 9 - 11 anos de estudos. Observou-se $80,2 \%$ residiam com o companheiro e $52,2 \%$ fora da população economicamente ativa. Verificou-se ainda que $67,61 \%$ pertenciam à classe econômica "C" (Tabela 1).

Nas variáveis referentes aos aspectos psicossociais das gestantes, observou-se: $24,46 \%$ com nível de estresse elevado, 40,84\% com nível de ansiedade moderado/intenso e 28,8\% (416 


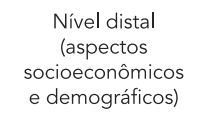

socioeconômicos
e demográficos)

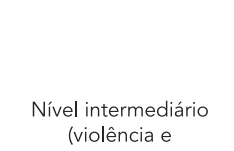

aspectos psicossocais)

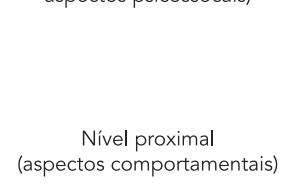

Desfecho
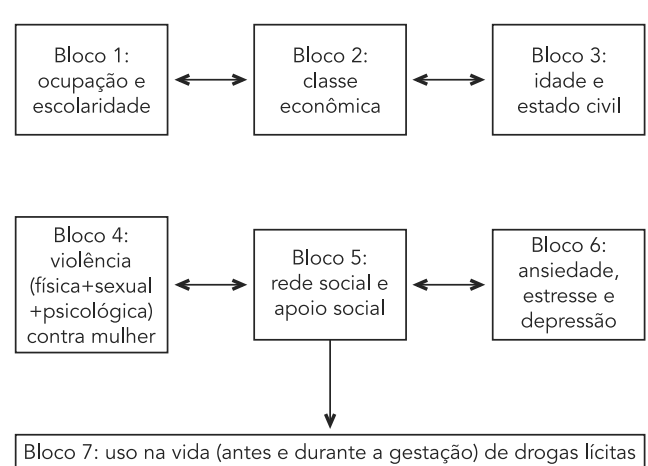
(bebidas alcóolicas e cigarro)

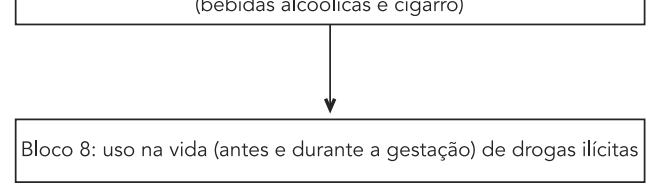

mulheres) com sintomas depressivos graves (Tabela 1).

A taxa de violência (psicológica e/ou física e/ou sexual) foi de $49,72 \%$. Sobre o suporte social observou-se $72,77 \%$ com ampla rede social e $65,21 \%$ com baixo apoio social, este compreendendo: apoio material e/ou apoio emocional e/ou apoio afetivo e/ou interação social positiva.

O uso de substâncias psicoativas na gestação foi de 22,32\% de bebidas alcóolicas - cerveja e/ ou vinho e/ou bebidas destiladas e $4,22 \%$ fizeram uso de cigarro, todas drogas lícitas. Acerca do consumo de drogas ilícitas - canabinoides e derivados e/ou cocaína e derivados, 1,45\% fez uso (Tabela 2).

Das 20 mulheres que utilizaram drogas ilícitas nos 3 meses anteriores à gestação, foi relatado o uso de: cocaína $(0,48 \%)$, maconha $(0,28 \%)$, merla $(0,21 \%)$, $\operatorname{crack}(0,21 \%)$, outras drogas $(0,55 \%)$. Nos 3 meses iniciais da gestação, foi relatado o consumo de: cocaína $(0,14 \%)$, maconha $(0,14 \%)$, merla $(0,07 \%)$, crack $(0,07 \%)$, outras drogas $(0,42 \%)$. A partir do $4^{\circ}$ mês de gestação, apenas 4 mulheres relataram o uso de drogas ilícitas.

$\mathrm{Na}$ análise logística múltipla hierarquizada, o uso de drogas ilícitas na gestação esteve, no nível distal, estatisticamente associado à monoparentalidade com OR = 2,62; IC95\%: 1,80-6,39 e valor de $\mathrm{p}=0,011$; e no nível intermediário, no nível elevado de estresse, $\mathrm{OR}=4,86$; IC95\%: 1,53-15,38 e valor de $\mathrm{p}=0,007$.
Observou-se também associação estatisticamente significante com todas as variáveis do nível proximal: uso de drogas lícitas na gestação. $\mathrm{O}$ uso de bebidas alcóolicas - cerveja e/ou vinho e/ ou bebidas destiladas esteve associado com OR = 4,4; IC95\%: 1,55-13,01 e valor de $\mathrm{p}<0,001$ e o uso de cigarro com OR = 6,7; IC95\%: 2,40-18,77 e valor de $\mathrm{p}<0,001$ (Tabela 3).

Não se observou associação estatisticamente significante entre o uso de drogas ilícitas na gestação e as seguintes variáveis no nível distal: idade, escolaridade, ocupação e classe econômica, e no nível intermediário: níveis de ansiedade, sintomas de depressão, violência sofrida e rede e apoio social.

\section{Discussão}

O estudo possibilitou identificar a taxa de uso de drogas ilícitas na gestação de 1,45\%. O uso de drogas ilícitas decresceu com o desenvolvimento da gestação. A análise hierarquizada mostrou associação com o uso de drogas lícitas, bebidas alcóolicas e cigarro, com nível alto de estresse e com situação de monoparentalidade.

O consumo de drogas ilícitas é um importante problema de saúde pública que carece de estudos epidemiológicos no Brasil, não sendo tarefa fácil observar a ocorrência deste desfecho em mulheres gestantes que estejam em 
Características socioeconômicas, demográficas e aspectos psicossociais das gestantes. São Luís, Maranhão, Brasil, 2010

\begin{tabular}{|c|c|c|}
\hline Variável & $\mathrm{n}$ & $\%$ \\
\hline \multicolumn{3}{|l|}{ Idade (anos) } \\
\hline Menos de 20 & 178 & 12,30 \\
\hline $20-34$ & 1.172 & 81,00 \\
\hline 35 ou mais & 97 & 6,70 \\
\hline \multicolumn{3}{|l|}{ Escolaridade (anos de estudo) } \\
\hline Menos de 9 & 181 & 12,53 \\
\hline $9-11$ & 1.091 & 75,55 \\
\hline 12 anos de estudos ou mais & 172 & 11,91 \\
\hline \multicolumn{3}{|l|}{ Reside com o companheiro } \\
\hline Sim & 1.160 & 80,17 \\
\hline Não & 287 & 19,83 \\
\hline \multicolumn{3}{|l|}{ Ocupação } \\
\hline Trabalha & 692 & 47,82 \\
\hline Nunca trabalhou & 755 & 52,18 \\
\hline \multicolumn{3}{|l|}{ Classe econômica } \\
\hline$A / B$ & 221 & 16,01 \\
\hline C & 933 & 67,61 \\
\hline$D / E$ & 226 & 16,38 \\
\hline \multicolumn{3}{|l|}{ Nível de estresse elevado * } \\
\hline Não & 1.093 & 75,54 \\
\hline Sim & 354 & 24,46 \\
\hline \multicolumn{3}{|l|}{ Nível de ansiedade } \\
\hline Normal/Leve & 856 & 59,16 \\
\hline Moderada/Intensa & 591 & 40,84 \\
\hline \multicolumn{3}{|l|}{ Sintomas depressivos graves } \\
\hline Sim & 416 & 28,80 \\
\hline Não & 1.026 & 71,20 \\
\hline \multicolumn{3}{|l|}{ Violência sofrida ** } \\
\hline Sim & 718 & 49,72 \\
\hline Não & 726 & 50,28 \\
\hline \multicolumn{3}{|l|}{ Rede social } \\
\hline Rede restrita & 394 & 27,23 \\
\hline Rede ampla & 1.053 & 72,77 \\
\hline \multicolumn{3}{|l|}{ 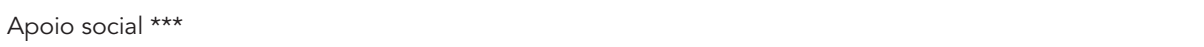 } \\
\hline Apoio baixo & 943 & 65,21 \\
\hline Apoio alto & 503 & 34,79 \\
\hline
\end{tabular}

* Estresse elevado: acima do último quartil (ponto de corte 30);

** Violência sofrida: violência psicológica + violência física + violência sexual;

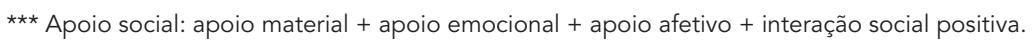

atendimento pré-natal, dada a baixa adesão ao atendimento, quer por questões individuais de vulnerabilidades das mulheres, quer por questões institucionais de dificuldade de acesso aos serviços 23 .

Outro fator importante relacionado à dificuldade de observação desse fenômeno refere-se à guarda dos filhos; a possibilidade de perda do poder familiar pela mãe, caso ela seja usuária dependente de drogas. A literatura internacional aponta barreiras: geográficas, organizacionais, econômicas, sociais e psicológicas - com possibilidade de sobreposição destas para as gestantes usuárias de 
Características do consumo de drogas entre as gestantes. São Luís, Maranhão, Brasil, 2010.

\begin{tabular}{|c|c|c|}
\hline Características & $\mathrm{n}$ & $\%$ \\
\hline \multicolumn{3}{|c|}{ Consumo de drogas lícitas } \\
\hline \multicolumn{3}{|c|}{ Bebidas alcóolicas * } \\
\hline Não & 1.124 & 77,68 \\
\hline Sim & 323 & 22,32 \\
\hline \multicolumn{3}{|l|}{ Cigarro } \\
\hline Não & 1.386 & 95,78 \\
\hline Sim & 61 & 4,22 \\
\hline \multicolumn{3}{|c|}{ Consumo de drogas ilícitas durante a gestação ** } \\
\hline Não & 1.424 & 98,55 \\
\hline Sim & 20 & 1,45 \\
\hline \multicolumn{3}{|c|}{3 meses antes da gestação } \\
\hline Maconha & 4 & 0,28 \\
\hline Merla & 3 & 0,21 \\
\hline Crack & 7 & 0,48 \\
\hline Cocaína & 3 & 0,21 \\
\hline Outras & 8 & 0,55 \\
\hline \multicolumn{3}{|c|}{3 primeiros meses da gestação } \\
\hline Maconha & 2 & 0,14 \\
\hline Merla & 1 & 0,07 \\
\hline Crack & 2 & 0,14 \\
\hline Cocaína & 1 & 0,07 \\
\hline Outras & 6 & 0,42 \\
\hline \multicolumn{3}{|c|}{ A partir do 4 o mês de gestação } \\
\hline Maconha & 1 & 0,07 \\
\hline Merla & - & - \\
\hline Crack & - & - \\
\hline Cocaína & - & - \\
\hline Outras & 3 & 0,21 \\
\hline
\end{tabular}

* Bebidas alcóolicas: cerveja + vinho + bebidas destiladas.

** Drogas ilícitas: maconha + cocaína + crack + merla + outras.

drogas acessarem os serviços de saúde, com destaque na barreira psicológica ao medo da mãe de perder a custódia do filho 24 . Ressaltase tanto na fonte citada quanto na legislação brasileira atual a possibilidade da perda da custódia do filho, observada no artigo 19 do Estatuto da Criança e do Adolescente (ECA) 25: "toda criança e adolescente tem direito a ser criado e educado no seio de sua família, e, excepcionalmente em família substitutiva, assegurada a convivência familiar e comunitária, em ambientelivredepessoasdependentesdesubstâncias entorpecentes".

A literatura recomenda o uso de testes biológicos para aferir com maior confiabilidade o consumo de drogas ilícitas, testes como os que utilizam urina, cabelo da gestante ou mesmo análise do mecônio do recém-nascido 26,27. Uma limitação desses testes é que não aferem informações em relação ao sincronismo do uso de drogas durante a gravidez e sobre a quantidade utilizada 28 .

Em estudos populacionais é importante pensar que o autorrelato é um método barato e prático para a identificação do uso de drogas, pois permite obter a sincronicidade de informações não disponíveis nos testes biológicos, além de permitir à mulher a possibilidade do relato mais verídico.

O estudo ter sido realizado com amostra de conveniência, cujo desenho é não probabilístico, e o objeto ser de baixa prevalência, diminuindo o poder dos testes, também são limitações deste trabalho. É também fator de limitação a amostra 
Análise hierarquizada ajustada por regressão logística múltipla dos fatores associados ao uso de drogas ilícitas na gestação. São Luís, Maranhão, Brasil, 2010.

\begin{tabular}{|c|c|c|c|c|c|c|}
\hline & \multicolumn{2}{|c|}{ Bloco 1} & \multicolumn{2}{|c|}{ Bloco 2} & \multicolumn{2}{|c|}{ Bloco 3} \\
\hline & OR (IC95\%) & Valor de $p$ & OR (IC95\%) & Valor de $p$ & OR (IC95\%) & Valor de $p$ \\
\hline \multicolumn{7}{|c|}{ Reside com o companheiro } \\
\hline Sim & 1,00 & & & & & \\
\hline Não & $2,62(1,80-6,39)$ & 0,011 & & & & \\
\hline \multicolumn{7}{|c|}{ Níveis de estresse elevado } \\
\hline Não & & & 1,00 & & & \\
\hline Sim & & & $4,86(1,53-15,38)$ & 0,007 & & \\
\hline \multicolumn{7}{|l|}{ Nível de ansiedade } \\
\hline Normal/Leve & & & 1,00 & & & \\
\hline Moderada/Intensa & & & $2,31(0,89-5,98)$ & 0,08 & & \\
\hline \multicolumn{7}{|l|}{ Violência sofrida } \\
\hline Não & & & 1,00 & & & \\
\hline Sim & & & $2,44(0,89-5,98)$ & 0,09 & & \\
\hline \multicolumn{7}{|c|}{ Consumo de bebida alcóolica } \\
\hline Não & & & & & 1,00 & \\
\hline Sim & & & & & $4,4(1,55-13,01)$ & $<0,001$ \\
\hline \multicolumn{7}{|l|}{ Consumo de fumo } \\
\hline Não & & & & & 1,00 & \\
\hline Sim & & & & & $6,7(2,40-18,77)$ & $<0,001$ \\
\hline
\end{tabular}

IC95\%: intervalo de 95\% de confiança; OR: odds ratio.

ter se restringido a mulheres com gravidez única, atendidas em pré-natal somente, e só até o quinto mês de gestação. No entanto, para além das questões implicadas na aferição da variável resposta, os dados observados - prevalência de $1,45 \%$ do uso de drogas ilícitas na gestação mostram-se, quando comparados aos da literatura nacional, próximos aos encontrados em estudos realizados no Brasil em 1999, em Porto Alegre, que estimou prevalência de 1,7\%, mas somente para cocaína 7, e em 2012, em Maringá, que estimou $0,51 \%$ para o uso de crack e $0,51 \%$ para maconha 10 .

Estudos estimam que uma porção considerável das gestantes que negam qualquer uso de substâncias psicoativas (30-50\%) apresenta testes biológicos positivos para estas 26 , fato observado também por Wendell 8 que sugere medo, culpa, vergonha, constrangimento e estigma das mulheres para relatarem tal acontecimento. O estudo brasileiro realizado por Siqueira 7, observou fatos semelhantes ao identificar o uso de cocaína durante a gestação em 1,7\% quando a técnica para aferição foram entrevistas, e em $6 \%$ quando aferiu a variável resposta por meio de análises toxicológicas, testes no mecônio.

É importante salientar que no presente trabalho observou-se que o consumo de drogas ilícitas na gestação decresce com o desenvolvimento do período gravídico das mulheres (Tabela 2).

Wendel 8 observa em seu estudo que existem múltiplos fatores de risco para o uso de drogas na gestação, incluindo dependência passada ou presente de qualquer droga, como o álcool e o tabaco, história de violência psicológica ou doença mental, história de abuso sexual ou físico, idade mais jovem, mais especificamente final da adolescência e início da década dos 20 anos, pressões ambientais, difícil acesso à informação e falta de conhecimento sobre os perigos do consumo de drogas ilícitas na gestação para o desenvolvimento fetal.

Já em estudo realizado por Morris 27, observase como fatores de risco para o abuso de substâncias durante a gravidez, a falta de apoio social, idade, história pessoal ou familiar de abuso de drogas, história pessoal de consumo de álcool e uso de tabaco, abuso sexual na infância, áreas geográficas, variações de etnias, níveis de escolaridade e nível socioeconômico.

Os fatores associados ao uso de drogas ilícitas na gestação observados por Wendell 8 e Morris 27 em estudos internacionais foram pesquisados neste estudo brasileiro, sendo que em São Luís o uso de drogas lícitas - álcool e cigarro - níveis alto de estresse e situação de monoparen- 
talidade apresentaram associação com a variável desfecho pesquisada, uso de drogas ilícitas na gestação.

Acerca do nível mais distal, o fato de a mulher gestante residir sem o companheiro mostrou-se, neste trabalho, como fator associado ao uso de drogas ilícitas. Tal situação foi também observada por Siqueira 7, que identificou que gestantes usuárias de cocaína apresentaram associação estatisticamente significativa com a situação de monoparentalidade e com menores condições socioeconômicas. Residir sem o companheiro pode ser entendido, no caso deste estudo, como situação de vulnerabilidade familiar, particularmente porque a literatura tem demonstrado que a saúde mental das mulheres no período gravídico passa por alterações que demandam atenção familiar ${ }^{2}$.

No nível intermediário, a variável nível de estresse elevado registrou associação estatisticamente significativa. Embora as variáveis nível de ansiedade moderada/alta e violência não tenham apresentado associação significante, registraram altos valores de OR. Provavelmente a baixa prevalência do evento influenciou a não associação, uma vez que outros estudos 29,30,31 associam violência ao uso de drogas ilícitas.

A gestação e o puerpério são períodos da vida da mulher que precisam ser acompanhados com especial atenção, pois envolvem inúmeras alterações físicas, hormonais, psiquiátricas e de inserção social, que podem refletir diretamente na saúde mental destas mulheres 32,33 . Nessa direção, o estudo aponta OR de 4,86 de mulheres com sintomas de estresse elevado e OR de 2,31 de mulheres com sintomas de ansiedade moderada e intensa. Sendo assim, sintomas de estresse elevado e de ansiedade apresentam-se como comorbidade e fator de risco para o uso de drogas ilícitas, conforme estudos apresentados por Pereira \& Lovisi 32 que defendem o período gravídico puerperal como fase de maior prevalência de transtornos mentais na mulher. É importante pensar também que usuários de drogas ilícitas podem apresentar maiores índices de sintomas de sofrimento psíquico, assim como saúde mental comprometida.

Estudo apresentado por Bailey et al. 34 apresenta associação entre violência e uso de drogas ilícitas na gestação. Observa-se que o uso de álcool e drogas ilícitas tem relação com violência entre parceiros íntimos e resultados adversos no recém-nascido. Os estudos têm descrito aumento do risco de uso de álcool e de substâncias ilícitas entre mulheres grávidas que sofrem algum tipo de agressão.

Outros trabalhos 29,30 apontam que mulheres vítimas de violência também foram mais pro- pensas a relatar o uso de álcool, e tendência a relatar níveis mais elevados de consumo de drogas ilícitas. O mesmo trabalho indica indícios de que as mulheres que sofrem violência podem automedicar-se com álcool, drogas ilícitas e medicamentos prescritos, a fim de lidar com o que é violento.

El-Bassel et al. 30 apresenta hipóteses que podem auxiliar na compreensão da associação entre violência e o uso de drogas ilícitas por mulheres gestantes, com base em diversos estudos qualitativos realizados com mulheres que sofreram abuso e violência. Como primeira hipótese, o estudo explica que as mulheres usuárias de drogas ilícitas são muitas vezes consideradas “sexualmente promíscuas". Essa percepção se justifica pela possibilidade de violação das normas tradicionais de gênero. A segunda, considera que as mulheres iniciam ou aumentam o uso de drogas ilícitas para lidar com as dificuldades do cotidiano, e observa que o uso de tranquilizantes ou de maconha foi citado como uma resposta de automedicação frequente para a dor física e emocional, vivida imediatamente após um episódio de violência. A terceira hipótese apresenta uma relação recíproca entre violência e abuso de drogas, em que abuso de drogas aumenta a violência, que aumenta a probabilidade do uso de drogas ilícitas.

Uma probabilidade duas vezes maior de ocorrência de uso de drogas ilícitas em mulheres que sofreram violência estimada neste estudo deve servir como alerta, e aponta para a necessidade de investigar a ocorrência destes dois eventos na população.

A maior parte dos pesquisadores em saúde mental concorda que há poucas diferenças entre as chamadas drogas lícitas e ilícitas, pois o impacto subjetivo sobre o indivíduo se torna irrelevante após certo tempo de consumo 31,35.

Pela especificidade da população em estudo, mulheres gestantes de gravidez não gemelar atendidas em pré-natal até o quinto mês de gestação, quaisquer formas e frequências de uso foram consideradas potencialmente prejudiciais. A assunção desse pressuposto não nega as orientações preconizadas pela OMS de que o uso de drogas não significa a presença de dependência química. No entanto, o consumo de drogas ilícitas pela mulher no período gravídico sinaliza a necessidade de uma investigação mais profunda sobre o tema e uma possível situação de vulnerabilidade que possa indicar a necessidade de intervenções precoces 36 .

Outra questão importante é que o uso simultâneo de drogas lícitas e ilícitas mostrou-se frequente. Estudos registram que há uma alta prevalência no poliuso de substâncias psicoati- 
vas, particularmente em usuárias com padrão de uso de dependência 36,37 . Essa realidade é semelhante à observada entre as gestantes estudadas, apesar da prevalência do uso de drogas lícitas ser maior do que a das drogas ilícitas 38 .

A maconha é a droga ilícita mais comumente consumida entre as mulheres gestantes, porém o uso desta droga durante a gestação é mais problemático. Um estudo qualitativo com 18 usuárias de crack observou que todas iniciaram o consumo de álcool e cigarro, fizeram uso pesado destas substâncias e em curto tempo chegaram às drogas ilícitas. Outro estudo quantitativo observou que fatores psicossociais como isolamento social e vulnerabilidade à violência urbana contribuem para a manutenção do consumo, enfatizando como os processos sociais e fatores contextuais têm participação determinante nos padrões de uso das drogas 39 .

Embora não seja possível esclarecer, neste trabalho, a relação causal entre uso de drogas lícitas e ilícitas durante a gestação, se o consumo de drogas lícitas levou ao uso de drogas ilícitas, ou se os fatores que propulsionam o consumo de drogas lícitas são os mesmos que levam ao uso de drogas ilícitas, este trabalho mostra que há forte associação, com significância estatística, entre o uso de drogas lícitas e ilícitas. É importante que outros estudos, com outros desenhos, investiguem a relação causal entre o uso de drogas lícitas e ilícitas.

No presente trabalho não observamos associação com classe econômica, fato que pode ser compreendido ao verificarmos que a literatura mostra a presença do uso de drogas em todas as classes socioeconômicas, em decorrência das mudanças observadas na cultura do consumo de drogas na contemporaneidade 39 .

Apesar de o presente estudo não detectar associação entre uso de drogas ilícitas com rede e apoio social, é importante lembrar que estudos como o de Morris 27 apresentaram o apoio social como fator de proteção ao consumo de drogas na gestação, sendo que Escriba-Aguir et al. 40 observa que as necessidades de apoio social são susceptíveis a mudanças durante as diferentes fases maternas (gravidez, parto e pós-parto) e que o nível de apoio disponível para as mulheres (estrutural e funcional) em resposta aos desafios da gravidez, parto e pós-parto e na vida pode ser relevante na determinação dos resultados de saúde.

O estudo estimou a prevalência do uso de drogas ilícitas na gestação em 1,45\% para a população em análise, com monoparentalidade evidenciada por residir sem o companheiro, apresentar níveis de estresse elevado e uso de drogas lícitas, álcool e cigarro, como fatores associados a este uso estatisticamente significativos.

Apesar de não ter sido observada associação estatisticamente significativa com nível de ansiedade e violência sofrida pela mulher para uso de drogas ilícitas na gestação, ambas apresentaram OR com altos valores. Esses valores mostraram as mulheres com nível de ansiedade entre moderado a intenso, e mulheres que sofreram violência tiveram chance dobrada de uso de drogas na gestação.

O estudo observou que o uso de drogas ilícitas na gestação decresce com a progressão da gravidez das mulheres e que as drogas mais consumidas foram as lícitas (álcool e cigarro), seguidas das ilícitas (canabinoides e derivados e/ou cocaína e derivados).

A detecção das gestantes com maior risco de uso de drogas durante o pré-natal pode permitir uma intervenção mais precoce, redução de comportamentos de risco à saúde e melhoria da qualidade da assistência materna e infantil. Durante o pré-natal deve-se dar especial atenção a mulheres que relatam uso de drogas lícitas ou que residem sem o companheiro ou apresentam altos níveis de estresse, pois estas têm maior risco de uso de drogas ilícitas. 


\section{Colaboradores}

P. C. Rocha, M. T. S. S. B. Alves, D. C. Chagas participaram de todas as etapas da elaboração do artigo. A. A. M. Silva, R. F. L. Batista e R. A. Silva participaram da concepção e redação final do artigo.

\section{Agradecimentos}

Agradecimentos às Fundações de Amparo à Pesquisa do Maranhão (FAPEMA) e de São Paulo (FAPESP) pelo patrocínio e viabilização da pesquisa. Agradecimentos às gestantes que compõem o estudo de coorte BRISA, aqui especificamente as que residem no Município de São Luís.

\section{Referências}

1. Moreira MM, Mitsuhiro SS, Ribeiro M. O consumo de crack na gestação. In: Ribeiro M, Laranjeira R, organizadores. O tratamento do usuário de crack. Porto Alegre: Editora Artmed; 2012. p. 548-65.

2. Camacho RS, Cantinelli FS, Ribeiro CS, Cantilino A, Gonsales BK, Braguittoni E, et al. Transtornos psiquiátricos na gestação e no puerpério: classificação, diagnóstico e tratamento. Arch Clin Psychiatry (São Paulo, Impr.) 2006; 33:92-102.

3. Betiol H, Barbiere MA, Silva AAM. Epidemiologia do nascimento pré-termo: tendências atuais. Rev Bras Ginecol Obstet 2010; 32:57-60.

4. Alarcon S. Drogas psicoativas: classificação e bulário das principais drogas. In: Alarcon S, organizador. Álcool e outras drogas: diálogos sobre um mal-estar contemporâneo. Rio de Janeiro: Editora Fiocruz; 2012. p. 103-29.

5. Costa MTZ, Quintal VS, Haro FMB. Drogas de abuso na gestação: as orientações no pré-natal são suficientes? Pediatria (São Paulo) 1998; 20:317-22.

6. Yamaguchi ET, Cardoso MMSC, Torres MLA, Andrade AG. Drogas de abuso e gravidez. Arch Clin Psychiatry (São Paulo, Impr.) 2008; 35 Suppl 1:44-7.
7. Siqueira LP, Fabri ACOC, Fabri RL. Aspectos gerais, farmacológicos e toxicológicos da cocaína e seus efeitos na gestação. Revista Eletrônica de Farmácia 2011; VIII:75-87.

8. Wendell AD. Overview and epidemiology substance abuse in pregnancy. Clin Obstet Gynecol 2013; 56:91-6.

9. Cunha GB. Exposição pré-natal à cocaína e efeitos comportamentais no recém-nascido [Tese de Doutorado]. Porto Alegre: Universidade Federal do Rio Grande do Sul; 2007.

10. Kassada DS, Marcon SS, Pagliarini MA, Rossi RM. Prevalência do uso de drogas de abuso por gestantes. Acta Paul Enferm 2013; 26:467-71.

11. World Health Organization. Multi-country study of women's health and domestic violence against women: summary report of initial results on prevalence, health outcomes and womens responses. http://www.who.int/gender/violence/who_mul ticountry_study/summary_report/summary_re port_English2.pdf (acessado em 23/Out/2010). 
12. Ribeiro MRC, Alves MTSSB, Batista RFL, Ribeiro CCC, Schraiber LB, Barbieri MA, et al. Confirmatory factor analysis of the WHO violence against women instrument in pregnant women: results from the BRISA Prenatal Cohort. PLoS ONE 2014; 9:e115382.

13. Griep RH, Chor D, Faersteins E, Lopes C. Confiabilidade teste-reteste de aspectos da rede social do Estudo Pró-Saúde. Rev Saúde Pública 2003; 37:379-85.

14. Li D, Liu L, Odouli R. Presence of depressive symptoms during early pregnancy and the risk of preterm delivery: a prospective cohort study. Hum Reprod 2009; 24:146-53.

15. Gonçalves B, Fagulha T. The Portuguese version of the Center for Epidemiologic Studies Depression Scale (CES-D). Eur J Psychol Assess 2004; 20:33948.

16. Radloff LS. The CES-D Scale: a self-report depression scale for research in the general population. Appl Psychol Meas 1977; 1:385-401.

17. Field T. Prenatal depression effects on early development: a review. Infant Behav Dev 2011; 34:1-14.

18. Cunha JA. Manual da versão em português das Escalas de Beck. São Paulo: Editora Casa do Psicólogo; 2001.

19. Beck AT, Epstein N, Brown G, Steer RA. An inventory for measuring clinical anxiety: psychometric properties. J Consult Clin Psychol 1988; 56:893-7.

20. Beck NC, Siegel LJ, Davidson NP, Kormeier S, Breitenstein A, Hall DG. The prediction of pregnancy outcome: maternal preparation, anxiety and attitudinal sets. J Psychosom Res 1980; 24:343-51.

21. Cohen S, Kamarck T, Mermelstein R. A global measure of perceived stress. J Health Soc Behav 1983; 24:385-96.

22. Luft CD, Sanches SO, Mazo GZ, Andrade A. Versão brasileira da Escala de Estresse Percebido: tradução e validação para idosos. Rev Saúde Pública 2007; 41:606-15.

23. Leodoiro E, González-Colmenero E, ConcheiroGuisán A, Castro A, Cruz A, López-Rivadulla M, et al. Maternal hair analysis for the detection of illicit drugs, medicines, and alcohol exposure during pregnancy. Ther Drug Monit 2013; 35:296-304.

24. Sexton RL, Carlson RG, Leukefeld CG, Booth BM. Barries to formal drug abuse treatment in the rural south: a preliminary ethnographic assessment. J Psychoactive Drugs 2008; 40:121-9.

25. Brasil. Lei no 8.069, de 13 de julho de 1990. Dispõem sobre o Estatuto da Criança e do Adolescente e dá outras providências. http://portal.mec.gov. br/seesp/arquivos/pdf/lei8069_02.pdf (acessado em 08/Jan/2016).

26. Hepburn M. Substance abuse in pregnancy. Curr Obstet Gynaecol 2004; 14:419-25.

27. Morris T. Economic status and substance abuse during pregnancy. Beckley: School of Graduate Nursing, Mountain State University; 2012.
28. Benhnke M, Smith VC. Prenatal substance abuse: short- and long-term effects on the exposed fetus. Pediatrics 2013; 131:e1009.

29. Sapori LF, Sena LL. Crack e violência urbana. In: Ribeiro M, Laranjeira R, organizadores. O tratamento do usuário de crack. Porto Alegre: Editora Artmed; 2012. p. 74-91.

30. El-Bassel N, Gilbert L, Wu E, Go H, Hill J. Relationship between drug abuse and intimate partner violence: a longitudinal study among women receiving methadone. Am J Public Health 2005; 95:46570 .

31. Bailey BA. Partner violence during pregnancy: prevalence, effects, screening, and management. Int J Womens Health 2010; 2:183-97.

32. Pereira PK, Lovisi GM. Prevalência da depressão gestacional e fatores associados. Arch Clin Psychiatry (São Paulo, Impr.) 2008; 35:144-53.

33. Bennett HA, Einarson A, Taddio A, Koren G, Einarson TR. Prevalence of depression during pregnancy: systematic review. Obstet Gynecol 2004; 103:698-709.

34. Bailey B, McCook JG, Hodge A, McGrady L. Infant birth outcomes among substance using women: why quitting smoking during preganancy is just as important as quitting illicit drug use. Matern Child Health J 2012; 16:414-22.

35. Hurt H, Betancourt LM, Malmud EK, Shera DM, Giannetta JM, Brodsky NL, et al. Children with and without gestational cocaine exposure: a neurocognitive systems analysis. Neurotoxicol Teratol 2009; 31:334-41.

36. Ribeiro M, Yamaguchi S, Duailibi LB. Avaliação de fatores de risco e proteção. In: Ribeiro M, Laranjeira $\mathrm{R}$, organizadores. $\mathrm{O}$ tratamento do usuário de crack. Porto Alegre: Editora Artmed; 2012. p. 226-38.

37. Ribeiro M. Avaliação psiquiátrica e comorbidade. In: Ribeiro M, Laranjeira R, organizadores. O tratamento do usuário de crack. Porto Alegre: Editora Artmed; 2012. p. 239-50.

38. Pupo MC, Ribeiro M, Marques ACPR. Escalas de avaliação. In: Ribeiro M, Laranjeira R, organizadores. O tratamento do usuário de crack. Porto Alegre: Editora Artmed; 2012. p. 291-324.

39. Ribeiro LA, Nappo AS, Sanchez ZVDM. Aspectos socioculturais do uso de crack. In: Ribeiro M, Laranjeira $\mathrm{R}$, organizadores. O tratamento do usuário de crack. Porto Alegre: Editora Artmed; 2012. p. 50-6.

40. Escriba-Aguir V, Royo-Marques M, Artazcoz L, Romito P, Perez IR. Longitudinal study of depression and health status in pregnant women: incidence, course and predict factors. Eur Arch Psychiatry Clin Neurosci 2013; 263:143-51.

41. Abeldaño RA, Fernández AR, Ventura CAA, Estario JC. Consumo de sustancias psicoactivas en dos regiones argentinas y su relación con indicadores de pobreza. Cad Saúde Pública 2013; 29:899-908. 


\section{Resumo}

This study analyzes the prevalence of illicit drug use and associated factors during pregnancy. This was a cross-sectional study of participants in the BRISA prenatal care cohort. Frequencies and hierarchical logistic regression were used. Estimated prevalence rates were $1.45 \%$ for illicit drug use, $22.32 \%$ for alcohol consumption, and $4.22 \%$ for smoking. The study population was mostly young (81\% in the 20-34-year bracket), with 9 to 11 years of schooling (75.55\%), with more than half of the women outside the workforce (52.18\%), and in economic class " $\mathrm{C}$ " (67.61\%). Pregnant women showed a high level of stress (24.46\%), moderate to intense anxiety (40.84\%), and severe depressive symptoms (28.8\%). Approximately half (49.72\%) of the pregnant women reported some type of violence, and they had wide networks (72.77\%) and low social support (65.21\%). Use of legal drugs, high stress levels, and single parenthood were independently associated with illicit drug use in pregnancy.

Pregnancy; Street Drugs; Risk Factors

\section{Resumen}

El artículo tiene como meta analizar la prevalencia y factores asociados con el consumo de drogas ilícitas durante embarazo. El estudio es de carácter transversal y se centra en el ámbito prenatal del proyecto BRISA cohorte. Utiliza medidas de frecuencia, intervalo de confianza y modelo de regresión logística múltiple jerárquica. La prevalencia estimada fue de $1.45 \%$ para uso de drogas ilícitas; 22,32\% para uso de alcohol y 4,22\% para consumo de cigarrillos. La población es joven, 20-34 años (81\%), la escolarización de 9 a 11 años de educación (75.55\%), con más de la mitad de las mujeres fuera de la población económicamente activa (52.18 \%) y clase social "C" (67,61\%). Las mujeres embarazadas tienen un alto nivel de estrés $(24,46 \%)$, sintomas de ansiedad de moderada a severa (40.84\%) y sintomas depresivos severos (28,8\%). Aproximadamente la mitad (49,72\%) de las mujeres reportó haber experimentado alguna forma de violencia; la mayoría cuenta con una gran red social $(72,77 \%)$ y bajo apoyo social (65,21\%). Drogas lícitas, altos niveles de estrés y madres solteras fueron los factores que se asociaron con significación estadística.

Embarazo; Drogas Ilícitas; Factores de Riesgo
Recebido em 20/Jan/2014

Versão final reapresentada em 04/Jun/2015 Aprovado em 10/Jun/2015 